\begin{tabular}{|c|c|}
\hline & $\begin{array}{l}\text { International Journal of Trend in Scientific } \\
\text { Research and Development (IJTSRD) }\end{array}$ \\
\hline 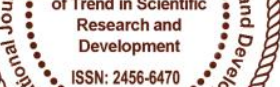 & International Open Access Journal \\
\hline 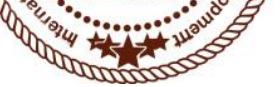 & ISSN No: 2456 - 6470 | www.ijtsrd.com | Volume - 2 | Issue -1 \\
\hline
\end{tabular}

\title{
A Study of Fusing Subjectivity and Distinct Issues in Data Mining
}

\author{
Sweta Kaushik \\ Student M.Tech (CSE), Monad University
}

\author{
Dr. Rajiv Singh \\ HOD (CSE), Monad University
}

\begin{abstract}
The world now is overpowered with data, the computerized upheaval has made digitized data simple to catch, process, store, disperse and transmit. The measure of data appears to continue forever expanding and the advance in computerized data procurement and capacity innovation has brought about the development of immense databases. The knowledge Discovery from tremendous number of databases and gigantic volume of data is a test. Inside these masses of data lies concealed data of key significance.
\end{abstract}

Keywords: data mining, rule, data warehousing, database

\section{INTRODUCTION}

At the point when there are such huge numbers of trees, how would we make important inferences about the woods? The freshest answer is data mining, which is being utilized both to expand incomes and to decrease costs. The potential returns are huge. Inventive associations worldwide are as of now utilizing data mining to find and bid to higher-value clients, to reconfigure their item offerings to build deals, and to limit misfortunes because of mistake or misrepresentation. Data mining is a process that uses a variety of data analysis tools to discover patterns and relationships in data that may be used to make valid predictions. The first and simplest analytical step in data mining is to describe the data, summarize its statistical attributes (such as means and standard deviations), visually review it using charts and graphs, and look for potentially meaningful links among variables (such as values that often occur together). As emphasized in a later section, collecting, ploring and selecting the right data are critically important. But data description alone cannot provide an action plan. We must build a predictive model based on patterns determined from known results, and then test that model on results outside the original sample. A good model should never be confused with reality but it can be a useful guide to understanding business. The final step is to empirically verify the model. Data Mining is an attempt to make sense of the information explosion embedded in this huge volume of data [BS 2004]. Many people treat Data Mining as a synonym for another used term, i.e. KDD, or Knowledge Discovery in Databases.

\section{II .Challenges in Data Mining:}

Gathering the data for mining is hard process without anyone else as the progressing operations ordinary create huge and immense measure of data. Data Mining enables the end clients to remove intriguing business data or examples from expansive databases, and the bigger the volume of data that can be prepared by data mining systems, the more prominent the trust in the outcome [BS 2004 and PM]. Data mining procedure can be of at least one of the accompanying capacities, for example, classification rules, regression, time series analysis, prediction, clustering, summarization, association rules and sequence discovery. The quantity of producing rules would be high and just few of the found examples are of the enthusiasm to the end.

\section{Types of data mining:}

1. Relational database: till now most data are stored in relational database and relational database is one of the biggest resources of the data mining objects. As we know relational database is highly structured data repository, data are described by a set of attributes and 
stored in tables. Data mining on relational database mainly focus on discovering patterns and trends.

2. Transactional database: transactional database refers to the collection of transaction records, in most cases they are sales records. With the popularity of computer and e-commerce, massive transactional database are available now. Data mining on transactional database focuses on the mining of association rules, finding the correlation between items in the transaction records.

3. Spatial database: spatial databases usually contain not only traditional data but also location or geographic information about the corresponding data. Spatial association rules describe the relationship between one set of features and another set of features in a spatial database. Algorithms for mining spatial association rules are similar to association rule mining except consideration of spatial data, the predicates generation and rules generation processes are based on Apriori.

\section{Temporal and time-series database: it differs from} traditional transaction data, for each temporal data item the corresponding time related attributes is associated. Temporal association rules can be more useful and informative than basic association rules.

\section{Data Mining Vs Data Warehousing:}

Once in a while, the data to be mined is first removed from an endeavor data stockroom into a data mining database or data store. There is some honest to goodness advantage if to be mined data is currently part of a data distribution center. The issues of sanitizing data for a data stockroom and for data mining are on a very basic level the same as. If the data has recently been washed down for a data distribution center, by then it most likely won't require furthermore cleaning in order to be mined. The data mining database may be a canny instead of a physical subset of your data distribution center, gave that the data stockroom DBMS can reinforce the additional benefit solicitations of data mining. In case can't, by then we will be in a perfect circumstance with an alternate data mining database.

\section{Data Mining, Machine Learning and Statistics:}

Data mining exploits propels in the fields of artificial intelligence (AI) and insights. The two controls have been chipping away at issues of pattem acknowledgment and order. The two groups have made awesome commitments to the comprehension and use of neural nets and choice trees. Data mining does not supplant conventional factual strategies. Or maybe, it is an expansion of factual strategies that is to a limited extent the after-effect of a noteworthy change in the insights group. The improvement of most measurable procedures was, as of not long ago, in view of rich hypothesis and logical strategies that worked great on the humble measures of data being examined. The expanded energy of PCs and their lower cost, combined with the need to examine gigantic data sets with a large number of lines, have permitted the improvement of new methods in view of an animal power investigation of conceivable arrangements. New procedures incorporate generally late calculations like neural nets and choice trees, and new ways to deal with more seasoned calculations, for example, discriminant analysis. By prudence of conveying to tolerate the expanded PC control on the immense volumes of accessible data, these procedures can surmised any ftinctional shape or association on their own. Traditional measurable methods depend on the modeler to indicate the practical frame and collaborations. The key point is that data mining is the utilization of these and other AI and measurable procedures to basic business issues in a manner that makes these systems accessible to the talented knowledge worker and the prepared insights proficient. Data mining is a device for expanding the efficiency.

\section{Data Mining Techniques:}

(i) Classification: classification is the most commonly applied data mining technique. Classification is a method of categorizing or assigning class labels to a pattern set under the supervision of a teacher. Decision boundaries are generated to discriminate between patterns belonging to different classes. The patterns are initially partitioned into training and test sets, and the classifier is trained on the former. The test set is used to evaluate the generalization capability of the classifier. A decision tree classifier is one of the most widely used supervised leaming methods used for data exploration. It's easy to interpret and can be represented as if-thenelse pyjes It approximates a function by piecewise constant regions and does not require any prior knowledge of the data distribution.

(ii) Association: The task of association rule mining is to find certain association relationships among a set of objects (called items) in a database. The association relationships are described in association rules. Each rule has two measurements, support and confidence. Confidence is a measure of the rule's strength, while support corresponds to statistical significance. The task of discovering association rules was first introduced in 
1993 [AIS 93]. Originally, association rule mining is focused on market "basket data" which stores items purchased on a per-transaction basis. A typical example of an association rule on market "basket data" is that $70 \%$ of customers who purchase bread also purchase butter. Later, association rule mining is also extended to handle quantitative data.

\section{Motivation and Problem Definition:}

Knowledge Discovery of Databases (KDD) is the way toward removing already obscure however helpful and huge data from substantial huge volume of databases. Data Mining is a phase in the whole procedure of KDD which appHes a calculation to extricate fascinating examples. Generally, such calculations produce immense volume of examples. These examples must be assessed by utilizing intriguing quality measures to mirror the client prerequisites. Intriguing quality is characterized in various ways, (I) Objective measures (ii) Subjective measures. Target measures, for example, support and certainty separate significant examples in view of the structure of the examples, while subjective measures, for example, startling quality and curiosity mirror the client viewpoint. Target measures of intriguing quality may not feature the most imperative examples created by the data mining framework, subjective measures for the most part work by looking at the convictions of a client against the pattems found by the data mining calculation. It ought to be noticed that both target and subjective measures ought to be utilized to choose intriguing rules. Objective measures can be utilized as a sort of first channel while subjective measures can be utilized as a last channel to choose genuinely fascinating guidelines [ZXS]. investigation of conceivable arrangements. New strategies incorporate generally late calculations like neural nets and choice trees, and new ways to deal with more seasoned calculations, for example, discriminant investigation. By excellence of conveying to hold up under the expanded PC control on the gigantic volumes of accessible data, these systems can rough any ftinctional frame or collaboration on their own. Traditional factual procedures depend on the modeler to indicate the practical shape and connections. The key point is that data mining is the use of these and other AI and factual systems to regular business issues in a manner that makes these procedures accessible to the talented knowledge laborer and additionally the prepared insights professional. Data mining is an instrument for expanding the profitability. Identifying interesting rules fi*om a set of discovered rules is not a simple task because a rule could be interesting to one user but of no interesting to another. The interestingness of a rule is a subjective matter because it depends on the user's existing concepts and information about the domain and user's interest. In this work we introduce another measure of rule interestingness that is shocking rules and we propose an algorithm for incremental association rules mining that integrates shocking interestingness criterion during the process of building the model. One of the main features of the proposed approach is to capture the user background knowledge, which is monotonically augmented. The proposed algorithm makes use of interestingness measure as the basis of extracting interesting patterns. This important feature of the proposed algorithm is attractive and desirable in many real life applications as the volume of data keeps on growing and changing over the time and therefore the user background knowledge is monotonically augmented.

\section{Data Mining in Way Humans Evaluating:}

Vast databases of soccer statistics expose the limited way human observers rate performance and suggest how they can do significantly better. The way we evaluate the performance of other humans is one of the bigger mysteries of cognitive psychology. This process occurs continuously as we judge individuals' ability to do certain tasks, assessing everyone from electricians and bus drivers to accountants and politicians. The problem is that we have access to only a limited set of data about an individual's performance-some of it directly relevant, such as a taxi driver's driving record, but much of it irrelevant, such as the driver's sex. Indeed, the amount of information may be so vast that we are forced to decide using a small subset of it. How do those decisions get made? Today we get an answer of sorts thanks to the work of Luca Pappalardo at the University of Pisa in Italy and a few pals who have studied this problem in the sporting arena, where questions of performance are thrown into stark relief. Their work provides unique insight into the way we evaluate human performance and how this relates to objective measures. 


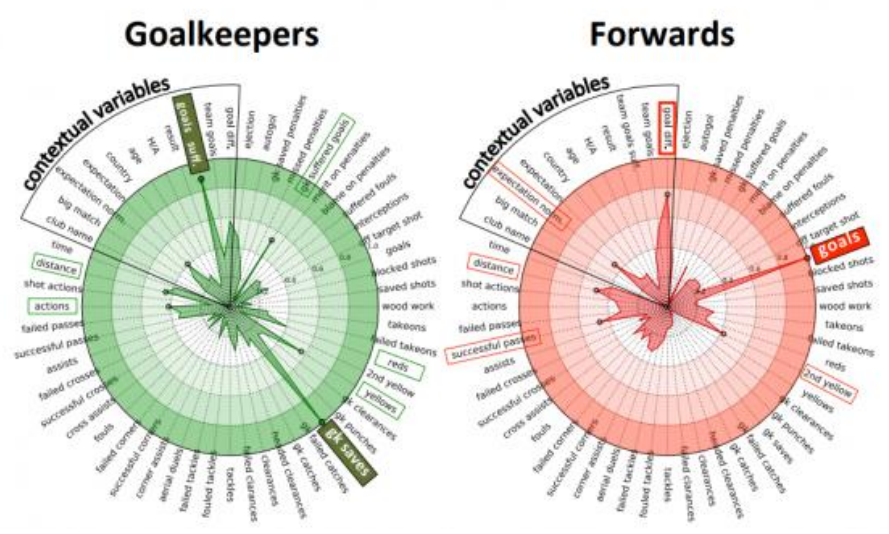

(Fig.1- The factors human observers use to rate performance are a small subset of objective measures)

Sporting performance is one area where detailed records of individual performance have been gathered for some years. Pappalardo and co focus on soccer, the world's most popular sport, and in particular on the performance of players competing at the top of the sport in Italy's Serie A football league.

\section{References}

1) [YAB 2007] Yafi, E., Alam, M.A., Biswas, R.: Development of Subjective

2) Measures of interestingness: From Unexpectedness to Shocking, Proceedings of

3) World Academy of Science, Engineering and Technology Volume 26 December 2007 ISSN 1307-6884.

4) YHAB 2009] Yafi, E., Al-Hegami, A. S., Alam, M.A., Biswas, R.: Incremental

5) Mining of Shocking Association Patterns, Proceedings of World Academy of

6) Science, Engineering and Technology, 49, January 2009
7) [AIS 93] R. Agrawal, T. Imielinski, A. Swami: "Mining Associations between

8) Sets of Items in Massive Databases", Proc. of the ACM-SIGMOD 1993 Int'1

9) Conference on Management of Data, Washington D.C, May 1993.

10) [AS 94] R. Agrawal, R. Srikant: "Fast Algorithms for Mining Association Rules",

11) Proc. of the 20th Int'l Conference on Very Large Databases, Santiago, Chile, Sept.1994.

12) [AS 96] R. Agrawal, J.C. Shafer; "Parallel Mining o f Association Rules", IEEE

13) Transactions on Knowledge and Data Engineering, Vol. 8, No. 6, December 1996,

14) [BA 99] R. J. Bayardo Jr. and R. Agrawal, "Mining the Most Interesting Rules"

15) In Proc. of the 5th ACM SIGKDD Int'l Conf. on Knowledge Discovery and Data

16) Mining, August 1999.

17) [BAG 99] R.J. Bayardo Jr., R. Agrawal, D. Gunopulos: "Constraint-Based Rule

18) Mining in Large, Dense Databases", Proc. of the 15 th Int'l Conf. on Data

19) Engineering, Sydney, Australia, March 1999

20) [CHY 96] Ming-Syan Chen, Jiawei Han and Philip S. Yu, Data Mining: An

21) Overview from a Database Perspective, IEEE Trans, on Knowledge and Data Engineering, Vol. 8, No. 6, Dec., 1996.

22) $\mathrm{CX}$ ] D. W. Cheung, Y. Xiao, Effect of Data Distribution in Parallel Mining of associations, Data Mining and Knowledge Discovery, Vol. 3, 1999. 